*This work has not yet been peer-reviewed"

*SI Appendix can be found here ${ }^{*}$

Social identity shapes antecedents and functional outcomes of moral emotion expression in online networks

\author{
William J. Brady ${ }^{1^{*}}$ \& Jay J. Van Bavel ${ }^{2,3}$ \\ ${ }^{1}$ Yale University, Department of Psychology \\ ${ }^{2}$ New York University, Department of Psychology \\ ${ }^{3}$ New York University, Center for Neural Science
}

\begin{abstract}
Author Note
${ }^{*}$ Correspondence concerning this article should be addressed to William Brady, Department of Psychology, Yale University. Contact: william.brady@yale.edu. This work was supported by the New York University Research Challenge Grant to JVB and a Postdoctoral Fellowship from the National Science Foundation to WJB \#1808868.
\end{abstract}

\title{
Contributions
}

WJB and JJVB designed research; WJB collected and analyzed data with input from JJVB; WJB wrote the paper and JJVB and WJB both contributed to revisions. 


\begin{abstract}
As social interactions increasingly occur through social media platforms, intergroup affective phenomena such as "outrage firestorms" and "cancel culture" have emerged with notable consequences for society. In this research, we examine how social identity shapes the antecedents and functional outcomes of moral emotion expression online. Across four pre-registered experiments $(N=1,712)$, we find robust evidence that the inclusion of moral-emotional expressions in political messages has a causal influence on intentions to share the messages on social media. We find that individual differences in the strength of partisan identification is a consistent predictor of sharing messages with moral-emotional expressions, but little evidence that brief manipulations of identity salience increased sharing. Negative moral emotion expression in social media messages also causes the message author to be perceived as more strongly identified among their partisan ingroup, but less open-minded and less worthy of conversation to outgroup members. These experiments highlight the role of social identity in affective phenomena in the digital age, and showcase how moral emotion expressions in online networks can serve ingroup reputation functions while at the same time hinder discourse between political groups.
\end{abstract}

Keywords: social identity, morality, emotion, politics, social networks 


\section{Social identity shapes the antecedents and functional outcomes of moral emotion expression in online networks}

On social media platforms, intergroup affective phenomena centered around morality such as viral moral outrage, public shaming and "cancel culture" are increasingly prevalent with notable consequences for individuals, groups and organizations (Ronson, 2015). For instance, recent work suggests that online moral rhetoric can precipitate violence in the real world (Mooijman et al., 2018), and examples such as the U.S. Capital riots showcase how moralized communications on social media are used as a tool to organize political violence (McEvoy, 2021). Understanding the psychological underpinnings of these intergroup moral behaviors in online networks may help shed light on the growing amount of intense animosity toward social outgroups in the United States (lyengar et al., 2019). This rise of intense partisan animosity and moralization on social media may even be contributing to partisan sectarianism (Allcott et al., 2020; Bail et al., 2018; Finkel et al., 2020). In the present research, we leverage Social Identity Theory (Tajfel \& Turner, 1979) to understand the antecedents and functional outcomes of sharing moral emotion expressions such as moral outrage online.

\section{Moral emotion expression in online networks}

In the digital age, social media has become one of the primary sources of moral outrage-outpacing traditional media, the news, and personal experience (Crockett, 2017). On social media platforms, people regularly use moral emotion expressions to 
signal, either to others or to the self, that something is relevant to the interests or good of society as defined by the conceptual knowledge of the expresser (Brady et al., 2020; Brady \& McLoughlin, 2021). For instance, moral outrage expression regarding corrupt behavior of a politician would be a canonical case of a moral emotion expression ("I am horrified and DISGUSTED by John Doe's speech!"), while expressing sadness due the death of a pet would not typically be considered a moral emotion expression ("I am so sad that my old cat passed away"). These expressions have significant consequences for online discourse.

In the context of online social networks, moral emotion expressions are robustly associated with increased sharing across multiple political topics (Brady et al., 2017), for political leaders during election periods (Brady et al., 2019), and for news articles (Valenzuela et al., 2017). This "moral contagion" phenomenon has been replicated using various methods for measuring moral expressions (Hopp et al., 2020) and in nonEnglish speaking countries (Ariztia, 2020) as well as across multiple social media platforms (Rathje et al., 2021).

Although the association between moral emotion expression and increased sharing in online networks is well-established with large samples of real-world behavior, this work has relied on observational data. As such, this body of work is unable to draw causal inferences about the spread of information. In the current paper, we present a series of pre-registered experiments to examine the causal influence of moral-emotional language on social media message sharing.

Furthermore, little work has empirically examined the psychological processes that explain why moral emotion expressions are likely to spread. Previous work found 
that group-based identity plays a key role in the spread of moral emotions online (Brady et al., 2017), such that people are more likely to share messages containing moralemotional expressions from ideological like-minded individuals. However, it is unclear if this is driven by social identity concerns or merely homophily (Brady et al., 2020). Here, we aimed to answer this question by examining how social identity processes help explain why people share political social media messages.

\section{A Social Identity Perspective}

One reason why moral emotion expression may spread widely on social media is because they are natural responses to fulfill group identity motivations that are highly salient on many social media platforms (Brady et al., 2020). With communications that are more likely to involve political ingroup members (Brady et al., 2017; Mosleh et al., 2021), political leaders disseminating partisan content (Brady et al., 2019; Wang \& Inbar, 2021), or the viral spread of political fake news (Lazer et al., 2018), social media is often a salient reminder of users partisan identities. This seems to be increasing over time, as more users are including their partisan identity in their social media profile pages (Rogers \& Jones, 2021). Furthermore, almost all social media users report that they see at least "a little" political content (94\% on Facebook and $89 \%$ on Twitter) when they log into their social media newsfeeds (Duggan \& Smith, 2016). As such, partisan identity is highly relevant to many social media users.

According to the Social Identity approach (Tajfel \& Turner, 1979; Turner et al., 1987), when group memberships are highly salient, people's individual identities become subsumed by group identity. As a consequence, their attitudes emotions and 
behaviors are influenced by evaluations made in terms of group, rather than individual goals (Abrams \& Hogg, 2004; Tajfel \& Turner, 1979). In this case, various group-based motivations arise including the need to belong in a group (Jetten et al., 2001) and maintaining positive distinctness between ingroup and outgroup members (Brewer, 1979; Tajfel \& Turner, 1979; Turner et al., 2007). Given the salience of partisan identity during a period of historically high polarization, it seems likely that partisan identity can have a strong influence on moralized social media discourse because these types of public expressions provide a clear cue to identity.

Following the social identity perspective, we hypothesized that group identity motives would influence the spread of moral emotion expressions on social media in terms of both antecedent conditions and functional outcomes. In terms of antecedent conditions, we reasoned that group categorization would predict the extent to which a person shares moral emotional messages either in terms of chronic individual differences (strength of identification with group) or due to specific contexts within the social media environment that make group identification more salient (e.g., exposure to contentious political news that threatens one's group identity). We therefore measured social identification and manipulated the salience of partisan identity.

In terms of functional outcomes, we reasoned that sharing moral emotion expressions function to enhance ingroup belonging by signaling that an individual is a strongly identified group member. For instance, previous work has examined how the expression of moral emotions serves as a signal of positive moral character broadly (Everett, Pizarro, \& Crockett, 2016; Jordan \& Rand, 2019), providing evidence that moral emotion expressions can increase the expressor's status among audience 
members. Here, we focus on audience evaluations specifically in terms of the identity of ingroup and outgroup members. If sharing moral emotion expressions can lead to positive ingroup evaluations, it may also provide a clear signal of intergroup differences and lead to negative evaluations by outgroup members (Barberá et al., 2015; Brady et al., 2017). Thus, we also proposed that expressing negative moral emotions can harm intergroup discourse.

\section{Overview}

Below, we present the results of four preregistered experiments that test the following hypotheses about the antecedents $(\mathrm{AH})$ of moral contagion. Individuals who are more strongly identified with their political group are more likely to share moral emotions in online social networks during political communications (AH1). When political group identities are made salient via identity-threatening or identity-affirming information, people will be more likely to share moral emotion expressions in online social networks during political communications (AH2). Studies 1-3 tested hypotheses $A H 1$ and $A H 2$ by measuring participants' intentions to share various messages that were manipulated to contain moral-emotional vs. neutral expressions. The messages were about various contentious topics in American politics or specific political events that were occurring around the time of data collection.

We then examined functional outcome (FO) hypotheses. People who share moral emotion expressions will be judged as more strongly identified with their political group than people who do not share moral emotion expressions (FO1). People who share moral emotion expressions will be judged by outgroup members as less open- 
minded than individuals who do not express moral emotions (FO2). Individuals who share moral emotion expressions will make outgroup members less interested in having a political conversation with them compared to people who do not share moral emotional expressions (FO3). Study 4 tested hypothesis FO1 - F03 by having participants make social judgments about message authors who shared tweets that were used as stimuli from Studies 1-3.

\section{Study 1}

Study 1 provided a test of whether moral-emotional expression in messages plays a causal role in decisions to share messages. This would extend previous correlational work on "moral contagion" that was conducted examining naturallyoccurring political conversations on Twitter (Brady et al., 2017, 2019). We also tested whether strength of identification with political groups would be associated with increased likelihood of sharing moral-emotional messages about politics $(A H 1)$. We report how we determined our sample size, all data exclusions (if any), all manipulations, and all measures in the study in our preregistration at https://osf.io/2nyq6.

\section{Method}

Participants. 360 Prolific workers were recruited to participate in a survey about social media and decision-making. After removing participants who did not complete the survey $(n=10)$ or did not complete the study in a reasonable timeframe (i.e., completed in less than 3 minutes; $n=0$ ), the final sample size was 350 . The mean age was 39.60 $(S D=13.14)$ and $56 \%$ of the sample $(n=196)$ was female. Our sample was 
approximately balanced on political party: $54 \%(n=188)$ identified as Republican and $46 \%(n=162)$ identified as Democrat. The mean political ideology was $0.09(S D=1.90)$ on a -3 (extremely liberal) to 3 (extremely conservative) scale, indicating a slight conservative skew. On average participants followed politics moderately closely $(M=$ $4.97, S D=1.54)$, based on a 1 (not closely at all) -7 (very closely) Likert scale. All participants were social media users (Twitter or Facebook), and $77 \%$ of participants used social media on a daily basis.

Measures. Identification with political party was measured using a 14-item scale that asked participants how much they agreed with statements regarding their identification with their party on a 1-7 Likert scale (Leach et al., 2008). Scale items showed excellent reliability (Cronbach's $\alpha=.95$ ) and were averaged together to form one composite measure of identification strength with their party, which was a preregistered decision $(M=5.12, S D=1.09)$.

Attitudes towards political topics were measured by asking participants to what extent they agree with a statement about gun control, President Trump, healthcare, immigration, and climate change on a 1 (do not at all agree) to 11 (agree completely) scale. For more details regarding attitude statements see SI Appendix, Section 1.1.

Procedure. The study was conducted in May, 2018. All studies described in the manuscript were approved under NYU IRB \#FY2016-301. Participants were told they were taking part in a survey about decision-making on social media. They completed demographics including a measure of identification with their political party before starting the main task. Participants were told the goal of the main task was to view pairs of messages and decide which message they would be more likely to share with their 
friends on social media. Prior work has found that self-reports of intentions to share messages on social media strongly predict real social media sharing (Mosleh et al., 2020).

Participants each viewed 5 trials of political social media messages that were aligned with attitudes of their self-reported political party including the following topics: gun control, President Trump, healthcare, immigration, and climate change. For instance, a Democratic participant saw messages that chastised Trump while a Republican participant saw messages that praised Trump. Crucially, on each trial the framing of the political messages was manipulated: each pair consisted of one message that expressed the topic attitude using moral-emotional language (e.g., "hate", "disgusting", "wrong") and the other expressed the same attitude using neutral language. On each trial, participants were asked to choose which message they would share with their friends on social media. We chose to have participants choose a message by clicking it to better simulate how people click buttons to share messages within their social media feed rather than answering a scale about their decision.

Stimuli. For each political party, we created one message pair per political topic, yielding 2 (Party: Democrat, Republican) x 2 (Language: moral-emotional, neutral) $\times 5$ (political topic) $=20$ total message stimuli. Fig. 1 displays an example of each type of stimulus. Stimuli for this study and all studies below are available at https://osf.io/jm3gn/. Messages that appeared together in pairs were matched closely on length, fluency and how 'weird' they seemed in pilot testing (to test whether some messages were more awkward or out of place than others). Pilot testing also confirmed that moral-emotional messages were perceived to be more emotional and related to 
morality than neutral messages, see SI Appendix, Section 1.2 for more details of pilot testing results.

Republican

2. Follow

Moral-emotional

The hatred spewed by Liberals about their current president is wrong. \#Trump $\leftarrow$ Reply 27 Retweet $\star$ Favorite $\cdots$ More

\section{Follow}

Neutral

The things that Liberals say about their current president are naive. \#Trump

$\leftrightarrow$ Reply 2 Z Retweet $\star$ Favorite. .0 More
Democrat

2. Follow

The hatred spewed by Trump on social media and in his speeches is wrong for a \#President of the U.S.

\& Reply 27 Retweet $\star$ Favorite..$\bullet$ More

2. 5 Follow

The rhetoric spoken by Trump on social media and in his speeches is strange for a \#President of the U.S.

\& Reply 27 Retweet $\star$ Favorite

Fig. 1. Examples of political messages containing moral-emotional expression vs. neutral expression for both political parties. The messages were virtually identical within each party except for a few moral-emotional words.

\section{Results}

In order to test our hypotheses, we conducted a mixed effects logistic regression model with trials nested within participants using the 'Imer' package in $R$ 3.6.1. In the results that follow we report results of a random intercept model as well as a model that treats stimulus as a random effect to ensure results can generalize beyond the specific stimuli used in the trials (Judd et al., 2012; Yarkoni, 2019). All models estimate the probability of choosing a moral-emotional message compared to a neutral message. Deidentified data and $R$ scripts for all analyses conducted are available at

\section{https://osf.io/jm3gn/.}

Across all trials, participants reported being significantly more likely to share a moral-emotional political message than a neutral political message, odds-ratio $(\mathrm{OR})=$ 
$1.29, p<.001,95 \% \mathrm{Cl}=[1.12,1.49]$. Thus, participants were $29 \%$ more likely to choose a moral-emotional message to share than a neutral message across political topics.

This result was robust when modeling stimulus as a random effect, $\mathrm{OR}=1.31, p=.026$, $95 \% \mathrm{Cl}=[1.03,1.67]$. Interestingly, this effect size is very similar (albeit slightly larger) to the effect size we observed in a sample over 500,000 online political messages on Twitter (Brady et al., 2017). This suggests that moral-emotional language has a causal influence on sharing. For exploration of political ideology and political party differences in sharing moral-emotional messages, see SI Appendix, Section 1.3.

Importantly, participants' strength of identification with their respective political party was a significant predictor of choosing the moral-emotional version of the message to share. Participants who were more strongly identified with their party were more likely to report that they would share moral-emotional messages, $\mathrm{OR}=1.20, p=$ $.007,95 \% \mathrm{Cl}=[1.05,1.37]$, see Fig. 2 . This result was robust when modeling stimulus as a random effect, $\mathrm{OR}=1.20, p=.007,95 \% \mathrm{Cl}=[1.05,1.36]$. It was also robust to statistically adjusting for each participants' self-reported attitudes towards the political topics, $\mathrm{OR}=1.19, p<.001,95 \% \mathrm{Cl}=[1.05,1.36]$, suggesting that participant's strength of identification with their political party predicts sharing of moral-emotional messages over and above strength of political attitudes. We did not find evidence for an interaction between political identity strength and political party, $\mathrm{OR}=1.09, p=.506,95 \% \mathrm{Cl}=$ $[0.84,1.41]$, suggesting that the association between identity strength is consistent across political parties. 


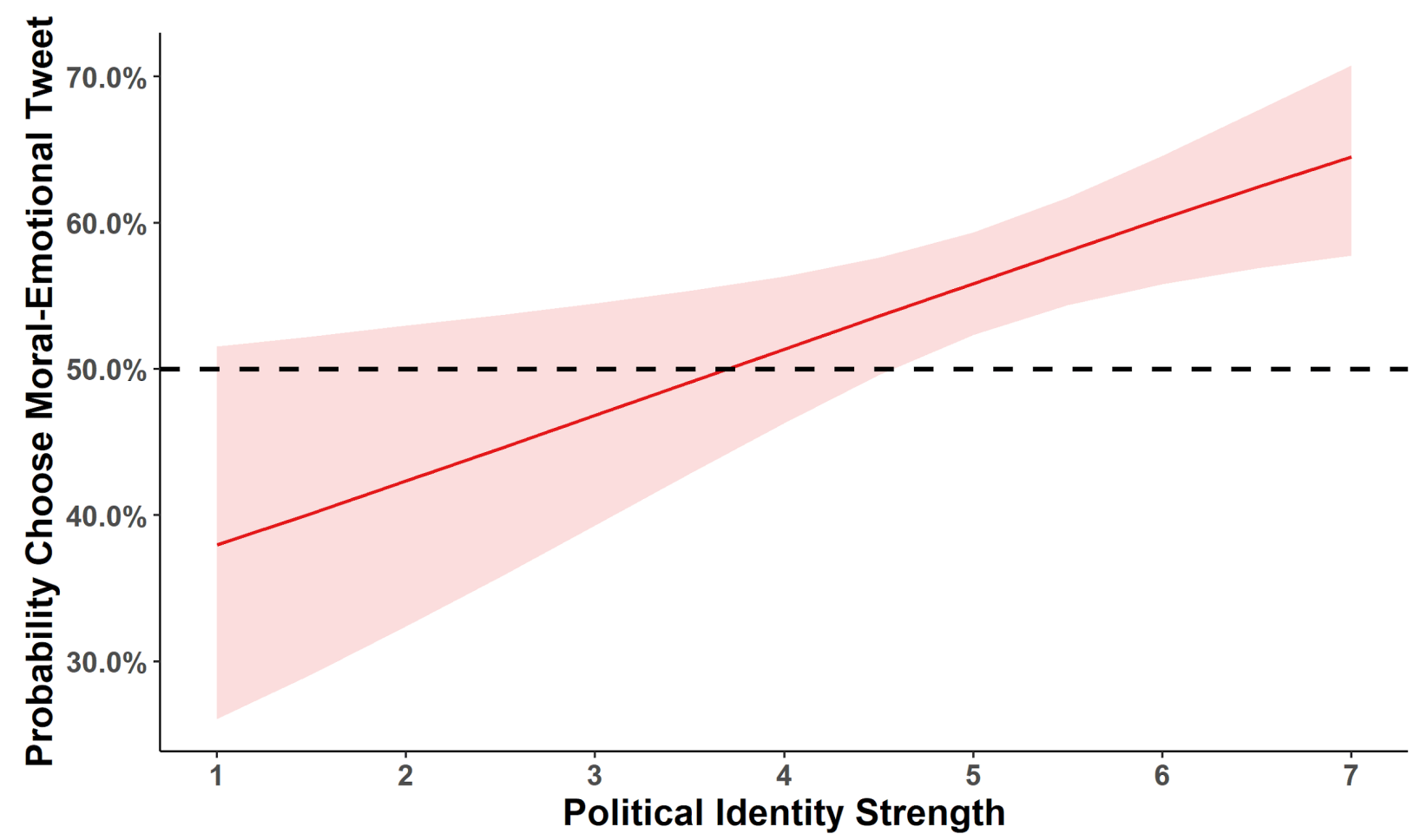

Fig. 2. The relationship between political identity strength on probability of choosing to share a moral-emotional political message across various contentious topics. Participants who were more strongly identified with the political group were significantly more likely to share negative moral-emotional messages about the political outgroup. The red line represents predicted values for each level of political identity strength generated from the fixed effect of the mixed model. Error bands represent $95 \%$ confident intervals for population-level predictions. The dotted line represents chance levels of choosing moral-emotional messages.

The results of Study 1 suggest that moral-emotional language causally impacts sharing intentions of political social media messages, especially among people who are the most strongly identified with their political party. These results support $A H 1$, suggesting that group identification may be an important predictor of moral emotion expression in online networks. 


\section{Study 2}

Study 2 sought to replicate the finding that identification with political parties is associated with increased likelihood of sharing negative moral-emotional messages about a political outgroup. Furthermore, we tested whether making political identity more salient with one-shot exposure to political information could increase sharing of moral-emotional language $(A H 2)$. To do so, we manipulated political group identity threat and affirmation in the context of the 2018 U.S. midterm elections by crafting news articles about the midterms. Finally, we examined the reasons people report for sharing moral-emotional vs. neutral content. We report how we determined our sample size, all data exclusions (if any), all manipulations, and all measures in the study in our preregistration at: https://osf.io/rh8ns.

\section{Method}

Participants. 600 Prolific workers participated in a survey about social media and decision-making. After removing participants who failed comprehension checks regarding the manipulation $(n=18)$ and who did not complete the survey $(n=20)$, the final sample size was 562 (mean age was $35.55[S D=12.36]$ and $59 \%$ of the sample [ $n$ $=329$ ] was female). $62 \%(n=351)$ identified as Democrat while $38 \%(n=211)$ identified as Republican. The mean political ideology was $-0.60(S D=1.85)$, indicating a slight liberal skew. On average participants followed politics moderately closely $(M=4.98, S D$ $=1.54$ ), all participants were social media users (Twitter or Facebook), and $77 \%$ of participants used social media on a daily basis. 
Measures. Identification with political party was measured using a single-item measure of identification strength (Postmes et al., 2013) which asked to participants to rate their agreement on a 1 (fully disagree) to 7 (fully agree) scale with the following statement: "I identify with the [Republican/Democrat] party" $(M=1.56, S D=1.33)$.

Attitudes towards political topics were measured by asking participants to what extent they agree with a statement about gun control, President Trump, healthcare, immigration, and climate change on a 1 (do not at all agree) to 11 (agree completely) scale, the same as in Study 1.

Procedure. The study was conducted in October, 2018 one week before the midterm elections in the U.S (November 6, 2018). Participants were invited to participate in a study about news consumption and decision-making. Participants completed demographic questionnaires and then were told they would view and be asked questions about three news articles. For each news article they viewed, participants answered a comprehension question, and then were asked which tweets they would be most likely to share among tweets related to the article they just read. To obscure the goal of the experiment, the first two news articles participants read were filler articles about entertainment and animals with headlines such as, "Cardi B and Post Malone won't compete for new artist Grammy". The third article was the "critical" article where participants were randomly assigned to view a political news article about the upcoming midterm election that was designed to either induce identity threat, identity affirmation, or neither (a neutral article). After reading each news article, participants viewed two trials of tweets afterwards and made a binary decision about which tweet to share. For the tweets that came after the two filler articles, participants chose between 
tweets expressing similar sentiments about the articles. For the tweets that came after the critical news article trials, we manipulated whether each tweet contained moralemotional or neutral language within a pair (see 'stimuli' below).

Manipulation of political identity threat. Participants were randomly assigned to view a critical third news article that either threatened their political identity, affirmed their political identity, or did neither (view a neutral article). To threaten Democrats' political identity (and to affirm Republicans' political identity), we created a news article ostensibly from the Associated Press that discussed key reasons why Democrats were likely to take heavy losses in the upcoming midterm which would allow Republicans to aggressively pass conservative legislation in the future. The article included real quotes from Democrat politicians regarding the importance of the upcoming elections. To threaten Republicans' political identity (and to affirm Democrats' political identity), we created an article ostensibly from the Associated Press that discussed key reasons why Republicans were likely to take heavy losses in the upcoming midterm which would allow Democrats to aggressively pass liberal legislation in the future. The article included real quotes from Republican politicians regarding the importance of the upcoming elections. The neutral article was about a lawsuit regarding a Lynyrd Skynyrd documentary. The three news articles that represented the manipulation can be viewed at https://osf.io/jm3gn/.

Stimuli. For the critical news article, we created two message pairs per political party, yielding 2 (Party: Democrat, Republican) x 2 (Language: moral-emotional, neutral) $\times 2$ (trials) for 8 total message stimuli. Each participant only viewed messages that corresponded to their self-reported political party. One trial consisted of tweets 
regarding the Brett Kavanaugh confirmation hearing, a highly controversial political event that occurred weeks before the study was conducted. The other trial consisted of messages about President Trump (the same used in Study 1). Fig. 3 displays the Brett Kavanaugh tweets.

Republican

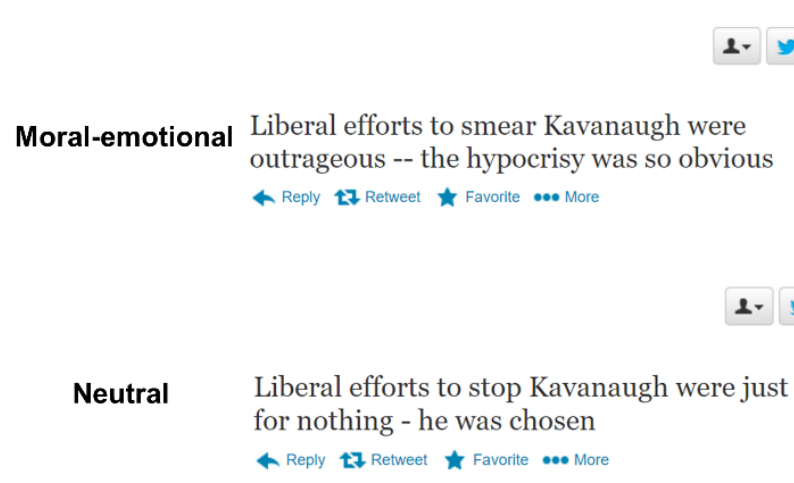

Democrat

2. Follow

It's disgusting that the \#GOP endorsed Brett Kavanaugh. He is unfit to be a Supreme Court judge

$\leftarrow$ Reply 27 Retweet $\downarrow$ Favorite

2. Follow

It's weird that the \#GOP endorsed Brett Kavanaugh. He is unqualified to be a Supreme Court judge

\& Reply 2 ? Retweet $\star$ Favorite $\cdots$ More

Fig. 3. Examples of political messages containing moral-emotional expression vs. neutral expression for both political parties.

\section{Results}

To test our hypotheses, we used the same models and analysis strategy as in Study 1. All models estimate the probability of choosing a moral-emotional message compared to a neutral message. Deidentified data and $R$ scripts for all analyses conducted are available at https://osf.io/jm3gn/. Unique to Study 2, we entered condition as a fixed effect with $k-1$ dummy coded variables where the reference group was the control group. We also statistically adjusted for the effect of political identity strength.

Replicating the causal effect of moral-emotional language on intentions to share. Across all conditions and trials, we found that moral-emotional messages were significantly more likely to be chosen for sharing, $\mathrm{OR}=4.10, p<.001,95 \% \mathrm{Cl}=[2.65$, 
6.30]. Moral-emotional messages were selected for sharing about $4 \mathrm{x}$ as often as neutral message. This effect was robust to modeling stimulus as a random effect, $\mathrm{OR}=4.10, p$ $<.001,95 \% \mathrm{Cl}=[2.66,6.30]$. For exploration of political ideology and political party differences in sharing moral-emotional messages, see SI Appendix, Section 2.2.

Replicating the results of Study 1, more strongly identified participants were significantly more likely to choose the moral-emotional version of the message to share, $\mathrm{OR}=1.60, p<.001,95 \% \mathrm{Cl}=[1.35,1.90]$, see Fig. 4 .

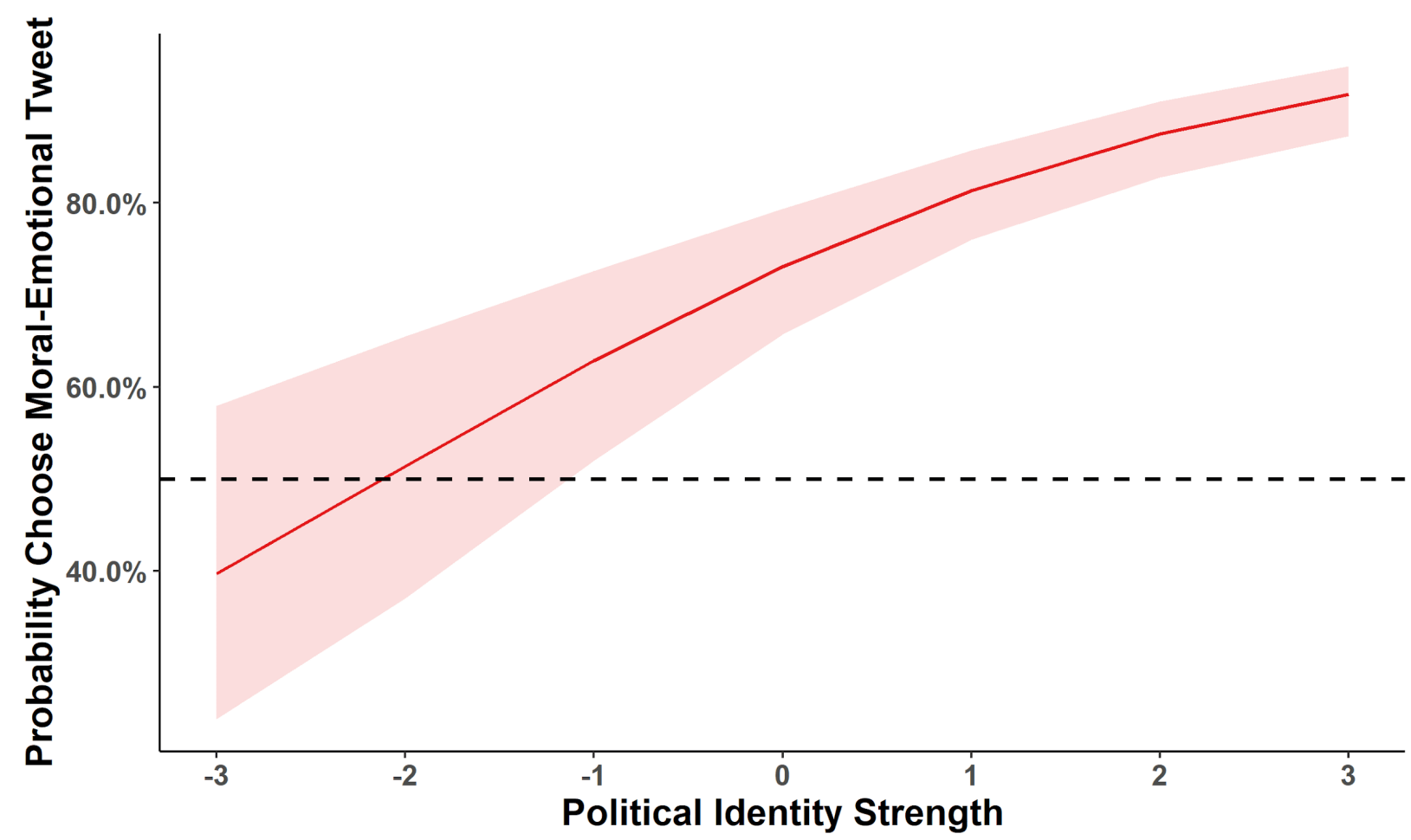

Fig. 4. The relationship between political identity strength on probability of choosing to share a moral-emotional political messages across various contentious topics. Participants who were more strongly identified with the political group were significantly more likely to share negative moral-emotional messages about the political outgroup. The dotted line represents chance levels of choosing moral-emotional messages.

We then examined if experimental manipulations of political identity changed moral contagion. As a manipulation check, we found that reading political articles in the 
threat condition produced significantly greater perceived group threat than the control condition $\left(M_{\text {diff }}=2.27, p<.001\right)$ and the affirm condition $\left(M_{\text {diff }}=2.32, p<.001\right)$, see also SI Appendix, Section 2.1. However, we found that inducing both identity threat and identity affirmation led to only marginal increases in choosing to share moral-emotional messages compared to the control condition, $\mathrm{OR}=1.60, p=.070,95 \% \mathrm{Cl}=[0.94$, 2.70], $\mathrm{OR}=1.60, p=.086,95 \% \mathrm{Cl}=[0.96,2.80]$, respectively. This pattern suggests that identity salience - rather than threat or affirmation specifically - might increase moral contagion. See SI Appendix, Section 2.3 for post-hoc analyses to test this possibility further.

In Study 2 we also explored participants' reported reasons for sharing moralemotional and neutral political messages. When participants choose to share a moralemotional message, $80 \%$ of the time they reported it was because the message represented their beliefs. In contrast, when participants choose to share a neutral message, only $46 \%$ of participants report it represents their beliefs. The percentages of each type of reason for sharing messages was significantly different among moralemotional and neutral messages, $X^{2}(4)=147.80, p<.001$. Table 1 displays all available reasons and corresponding frequencies. 


\begin{tabular}{lcc}
\hline \multicolumn{1}{c}{ Reason share } & Moral-emotional message & Neutral message \\
\hline It represents my beliefs & $80 \%$ & $46 \%$ \\
I want others to see the message & $10 \%$ & $13 \%$ \\
It feels good to share the message & $1 \%$ & $4 \%$ \\
I want to be funny or troll people & $1 \%$ & $3 \%$ \\
None of the above & $8 \%$ & $34 \%$
\end{tabular}

Table 1. Frequencies for reasons participants report sharing moral-emotional vs. neutral political messages.

The results of Study 2 replicated the causal influence of moral-emotional language on participants to decisions to share tweets and replicated the association between higher political group identification and decisions to share moral-emotional messages $(A H$ 1). Results did not discern whether making group identity threat salient vs. affirming group identity has a greater impact on moral-emotional message sharing. However, exploratory contrast analysis provided tentative evidence that when political group identity is made salient in political news either through either threat or affirmation, people are more likely to choose moral-emotional messages ( $A H 2)$. Furthermore, when participants intended to share moral-emotional messages, they claimed it was because it represented their beliefs significantly more than when participants shared a neutral message. Study 3 was designed to provide a confirmatory test of the effect of identity salience on decisions to share moral-emotional messages in order to further examine where $A H 3$ is supported. 


\section{Study 3}

Study 3 sought to further conceptually replicate the causal influence of moralemotional language on tweet sharing and the finding that strength of identification with political groups is associated with increased likelihood of sharing moral-emotional messages $(A H 1)$. We also sought to provide a confirmatory test of whether making political group identities salient is sufficient to increase sharing of moral-emotional messages $(A H 2)$. Finally, because we noticed such a large effect of choosing moralemotional messages in Study 2, we decided to make the moral-emotional content even more extreme to avoid ceiling effects of sharing (which we speculated comes along with the contentious political contexts in which we ran the previous studies). In other words, we aimed to make people less likely to share moral-emotional messages in general because it would require sharing more extreme language. Here, we focused on identity threat and identity affirmation in the context of the 2019 U.S. government shutdown. We report how we determined our sample size, all data exclusions (if any), all manipulations, and all measures in the study in our preregistration at:

\section{https://osf.io/tjvw6.}

\section{Method}

Participants. 600 Prolific workers were recruited to participate in a survey about social media and decision-making. After removing participants who failed comprehension checks regarding the manipulation $(n=23)$ and who did not complete the survey $(n=17)$, the final sample size was 560 (mean age was 32.64 [ $S D=12.08$ ] and $58 \%$ of the sample $[n=323$ ] was female. $69 \%(n=387)$ identified as Democrat. 
The mean political ideology was $-0.84(S D=1.62)$, indicating a slight liberal skew. On average participants followed politics moderately closely $(M=4.68, S D=1.49)$, all participants were social media users (Twitter or Facebook), and $79 \%$ of participants used social media on a daily basis. For further details on participant demographics including distributions, frequency tables and scale details, see SI Appendix, Study 3.

Measures. Identification with political party was measured as in Study 2 using a single-item measure of identification strength which asked to participants to rate their agreement on a 1 (fully disagree) to 7 (fully agree) scale with the following statement: "I identify with the [Republican/Democrat] party" $(M=1.62, S D=1.22)$.

Attitudes towards political topics were measured by asking participants to what extent they agree with a statement about gun control, President Trump, healthcare, immigration, and climate change on a 1 (do not at all agree) to 11 (agree completely) scale. For more details regarding attitude statements see SI Appendix, Study 3.

Procedure. The study was conducted in January, 2019 in the midst of the U.S. government shutdown. This time period was chosen because it was a contentious political time as each political party blamed one another for the endurance of the government shutdown (which costs many people jobs and income). The procedure was identical to Study 2 with the primary exception that the new articles focused on the government shutdown. After viewing each type of news article (2 filler and 1 critical article), participants viewed two trials of tweets and made a binary decision about which tweet to share. For the filler trials, tweets expressed various sentiments about the content of the articles. For the critical trials, we manipulated the tweets to contain either moral-emotional or neutral language within a pair (see 'stimuli' below). 
Manipulation of political identity threat. As in Study 3, participants were randomly assigned to view a critical third news article that either threatened their political identity, affirmed their political identity, or did neither. To threaten Democrats' political identity (and to affirm Republicans' political identity), we created a news article ostensibly from the Associated Press that discussed key reasons why Democrats were being blamed for the government shutdown by the public, and that Trump's image as a leader was being bolstered as a result. The article included real quotes from Democratic politicians referencing President Trump. By highlighting that Democrats being blamed for the political turmoil of the shutdown, the article was designed to make it seem as if the image of Democrats was taking a reputational hit across the U.S.

To threaten Republicans' political identity (and to affirm Democrats' political identity), we created an ostensibly from the Associated Press that discussed key reasons why Republicans were being blamed for the government shutdown by the public, and that Trump's image as a leader was being harmed as a result. The article included real quotes from Republican politicians regarding Democrat senators being the real people to blame. By highlighting that Republicans were being blamed for the political turmoil of the shutdown, the article was designed to make it seem as if the image of Republicans was taking a reputational hit across the U.S. The neutral article was about a lawsuit regarding a Lynyrd Skynyrd documentary as in Study 2. The three news articles that represented the manipulation can be viewed at https://osf.io/jm3gn/.

Stimuli. For the critical news article, we created two message pairs per political party, yielding 2 (party: Democrat, Republican) x 2 (message: moral-emotional, neutral) $x 2$ (trials) $=8$ total message stimuli. One trial consisted of tweets regarding the general 
disapproval of the political outgroup, and the other trial consisted of messages about President Trump (the same used in Study 1). Fig. 5 displays example tweets. All stimuli are available at https://osf.io/jm3gn/. Messages that appeared together in pairs were matched on length, fluency and how "weird" they seemed in pilot testing.

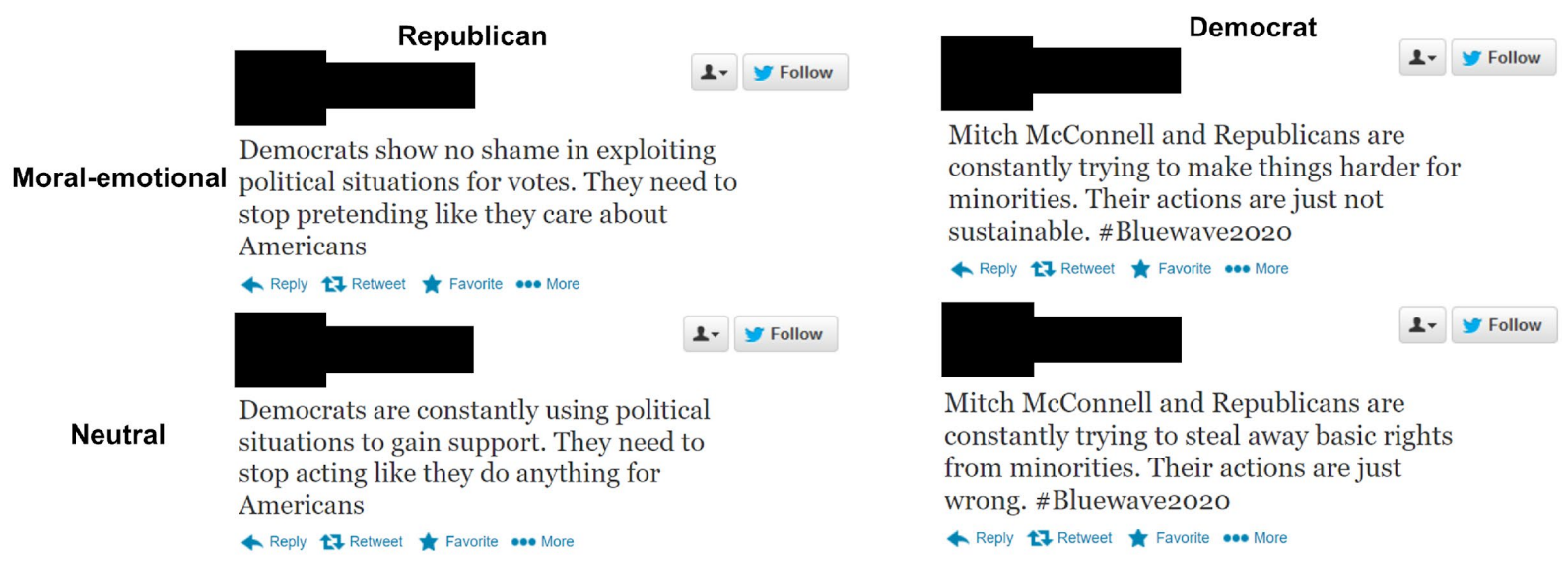

Fig. 5. Examples of political messages containing moral-emotional expression vs. neutral expression for both political parties.

\section{Results}

In order to test our hypotheses, we used the same models and analysis strategy as in Study 2. We again replicated the finding that moral-emotional messages were $57 \%$ more likely to be chosen for sharing, $\mathrm{OR}=1.57 p=.001,95 \% \mathrm{Cl}=[1.19,2.10]$. We also replicated prior findings for political identity strength such that more strongly identified participants were significantly more likely to choose the moral-emotional version of the message to share, $\mathrm{OR}=1.31, p<.001,95 \% \mathrm{Cl}=[1.15,1.50]$. Thus, moral-emotions are more likely to be shared--especially among highly identified group members. 
Regarding the identity threat and affirmation conditions, as a manipulation check we confirmed that reading articles in the threat condition indeed produced significantly greater perceived threat than the control condition $\left(M_{\text {diff }}=2.02, p<.001\right)$ and the affirm condition $\left(M_{\text {diff }}=1.18, p<.001\right)$. However, inducing identity threat and identity affirmation did not lead to significant differences in choosing to share moral-emotional messages compared to the control condition, $\mathrm{OR}=0.91, p=.347,95 \% \mathrm{Cl}=[0.57$, $1.20], \mathrm{OR}=1.60, p=.619,95 \% \mathrm{Cl}=[0.62,1.30]$, respectively. Collapsing the conditions to test the effect of salience of identity (a pre-registered decision) also did not reveal significant changes in choosing to share moral-emotional messages, $\mathrm{OR}=0.87$, $p=.410,95 \% \mathrm{Cl}=[0.62,1.20]$. Thus, Study 3 could not confirm that making group identity salient by reading political articles increased moral-emotional message selection. Condition did not interact with political identity strength in any of the models, see SI Appendix, Section 3.2.

\section{Study 4}

Study 4 investigated the functional outcomes of expressing outgroup-sanctioning moral-emotional messages on social media. Specifically, we tested whether expressing moral emotions makes the expresser viewed as more identified with their political ingroup (FO1). We also tested whether expressing moral emotions comes as a cost of outgroup relations (FO2-3). We report how we determined our sample size, all data exclusions (if any), all manipulations, and all measures in the study in our preregistration at: https://osf.io/kyfcx. 


\section{Method}

Participants. 240 Prolific workers were recruited to participate in a survey about social media and decision-making (mean age was $34.23[S D=13.70]$ and $60 \%$ of the sample [ $n=143]$ was female. All participants completed the task and all identified as Democrat or Republican and thus no participants were removed. 59\% $(n=142)$ identified as Democrat. The mean political ideology was $-0.57(S D=2.00)$, indicating a slight liberal skew. On average participants followed politics moderately closely $(M=$ 4.95, $S D=1.53$ ), all participants were social media users (Twitter or Facebook), and $80 \%$ of participants used social media on a daily basis.

Procedure. The study was conducted at the beginning of March, 2020 (prior to the Covid-19 pandemic affecting daily life in the United States). Participants were told that their task was to judge the extent to which expressors of social media messages identify with their political party. Participants viewed 5 messages for each trial type where we manipulated the message content to form a 2 (Language: moral-emotional, neutral) $\times 2$ (Party: ingroup, outgroup) within-subjects design. Thus, participants viewed a total of 20 messages. After viewing each social media message, participants were asked 4 questions: whether they thought the author was a Democrat or a Republican, how identified the author was with their political party, how open-minded the message author was, and how interested the participant would be to engage the message author in a political discussion.

Measures. The participant's attitudes towards political topics were measured by asking participants to what extent they agreed with a statement about gun control, 
President Trump, healthcare, immigration, and climate change on a 1 (do not at all agree) to 11 (agree completely) scale, the same as the previous studies.

On each trial, the message author's perceived political party (as judged by the participant) was measured as a binary choice (Democrat or Republican). The message author's perceived identification with their political party (as judged by participants) was measured on a 1 (Not at all identified) to 7 (Very identified) Likert scale. The message author's perceived open-mindedness was measured on a 1 (not at all open-minded) to 7 (very open-minded) Likert scale. The interest of the participant in having a political discussion with the message author was measured on a 1 (not at all interested) to 7 (very interested) Likert scale.

Stimuli. To form our 2 (language: moral-emotional, neutral) x 2 (party: ingroup / outgroup) within-subjects design, we used Democrat and Republican-leaning stimuli from the previous studies. An ingroup message was defined as a message expressing aligned-attitude message (i.e., if a Republican participant viewed a Republican-leaning message), whereas an outgroup message was defined as a message expressing a counter-attitudinal statement (i.e., if a Republican participant viewed a Democratlearning attitude message). In Study 4, participants a saw messages about the following political topics: gun control, abortion, healthcare, President Trump, Democrat/Republican policy.

\section{Results}

In order to test our hypotheses, using the 'Imer' package in $R$ 3.6.1 to we performed a 2 (language: moral-emotional, neutral) $\times 2$ (party: ingroup, outgroup) mixed 
model ANOVA on the following dependent variables: perceived group identification of the message author, perceived open-mindedness of the message author, and interest of the participant in having a political discussion with the message author. All analyses below correct for multiple comparisons for three dependent variables based on a Bonferroni correction of assuming an alpha of $.05 / 3=.017$.

We first examined whether expressing moral-emotional messages makes the expresser viewed as more identified with their political ingroup. Testing the effects of language and group on perceived identity strength revealed a significant main effect of language, $F(1,238)=291.60, p<.001, \eta^{2}{ }_{p}=.55$, such that both ingroup and outgroup message authors who used moral-emotional language $\left(M_{\text {ingroupME }}=5.89 S D=0.81\right.$, MoutgroupME $=6.03, S D=1.18)$ were perceived to be more strongly identified with their political group than those who used neutral messages $\left(M_{\text {ingroupN }}=5.20 S D=0.87\right.$, MoutgroupN $=5.29, S D=1.05$ ), see Fig. 6 . There was no significant main effect of group, $F(1,238)=3.43, p=.065, \eta^{2} p=.01$, such that the authors who used moral-emotional language were viewed as more strongly identified with their party regardless of whether they were ingroup or outgroup members. There was no interaction between language and group, $F(1,238)=0.65, p=.421, \eta^{2} p=.00$. The results support hypothesis $F O 1$, that expressing moral-emotional language makes message authors appear more identified with their respective group. 


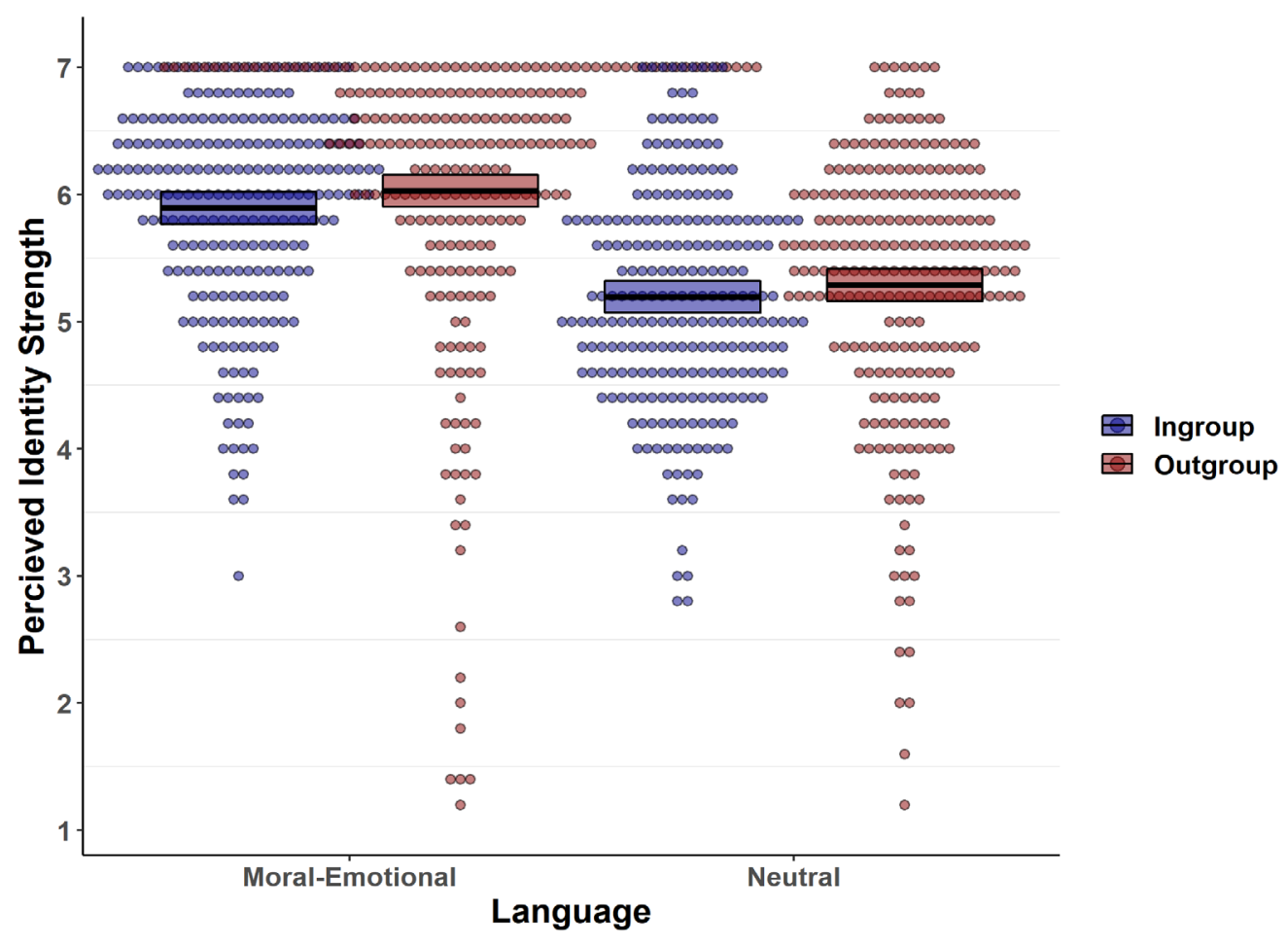

Fig. 6. The effect of language type and group on perceived political identity strength of a message author. Message authors who expressed moral-emotional language were perceived to be significantly more identified with their political party for both ingroup and outgroup members.

We also tested whether language and group affected indicators of intergroup relations. Testing the effects of language and group on perceived open-mindedness revealed a significant main effect of both language, $F(1,238)=244.40, p<.001, \eta^{2} p=$ .51 , and group, $F(1,238)=299.50, p<.001, \eta^{2} p=.56$. However, these main effects were qualified by a significant interaction, $F(1,238)=48.90, p<.001, \eta^{2} p=.17$ which revealed that the reduction in perceived open-mindedness from using moral-emotional language was significantly greater for outgroup members $\left(M_{\text {diff }}=-0.98\right)$ compared to ingroup members $\left(M_{\text {diff }}=-0.44\right)$, see Fig. 7. Outgroup members who used moral-emotional language showed the lowest perceived open-mindedness of any condition $(M=2.05)$. 


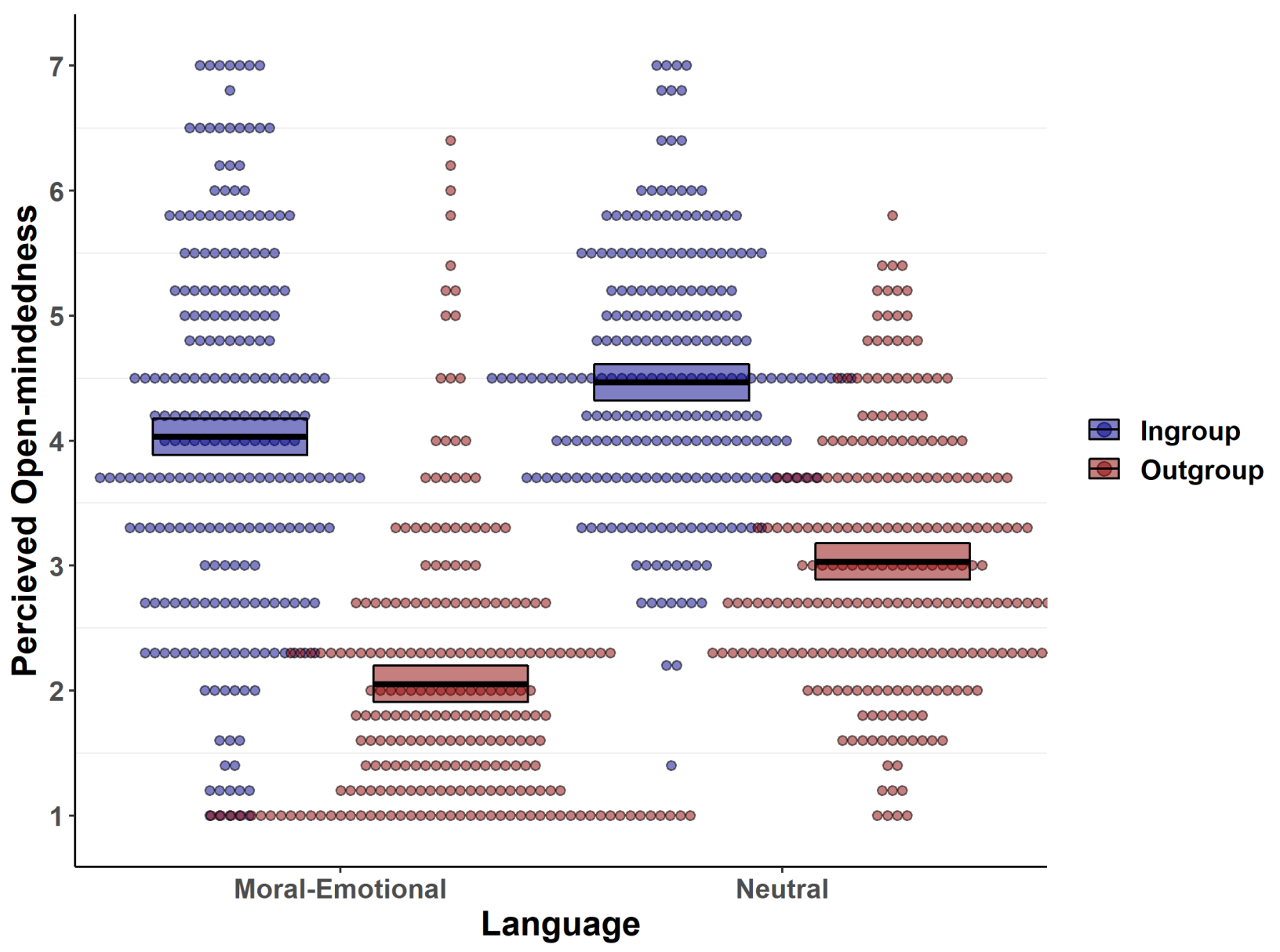

Fig. 7. The effect of language type and group on perceived open-mindedness of a message author. Message authors who expressed moral-emotional language were perceived to be significantly less open-minded, in particular if the author was a political outgroup member.

Testing the effects of language and group on interest in having a political discussion revealed a significant main effect of both language, $F(1,238)=102.40, p$ $<.001, \eta^{2} p=.30$, and group, $F(1,238)=255.50, p<.001, \eta^{2} p=.52$. However, these main effects were qualified by a significant interaction, $F(1,238)=19.63, p<.001, \eta^{2} p=.18$, which revealed that the reduction in interest in having a political discussion from expressing moral-emotional language compared to neutral language was significant for political outgroup members $\left(M_{\text {diff }}=-0.72, p<.001\right)$ but not ingroup members $\left(M_{\text {diff }}=-\right.$ $0.15, p=.064)$, see Fig. 8. Participants had the lowest interest in having a political 
discussion with outgroup members who expressed moral-emotional language $(M=$

2.11).

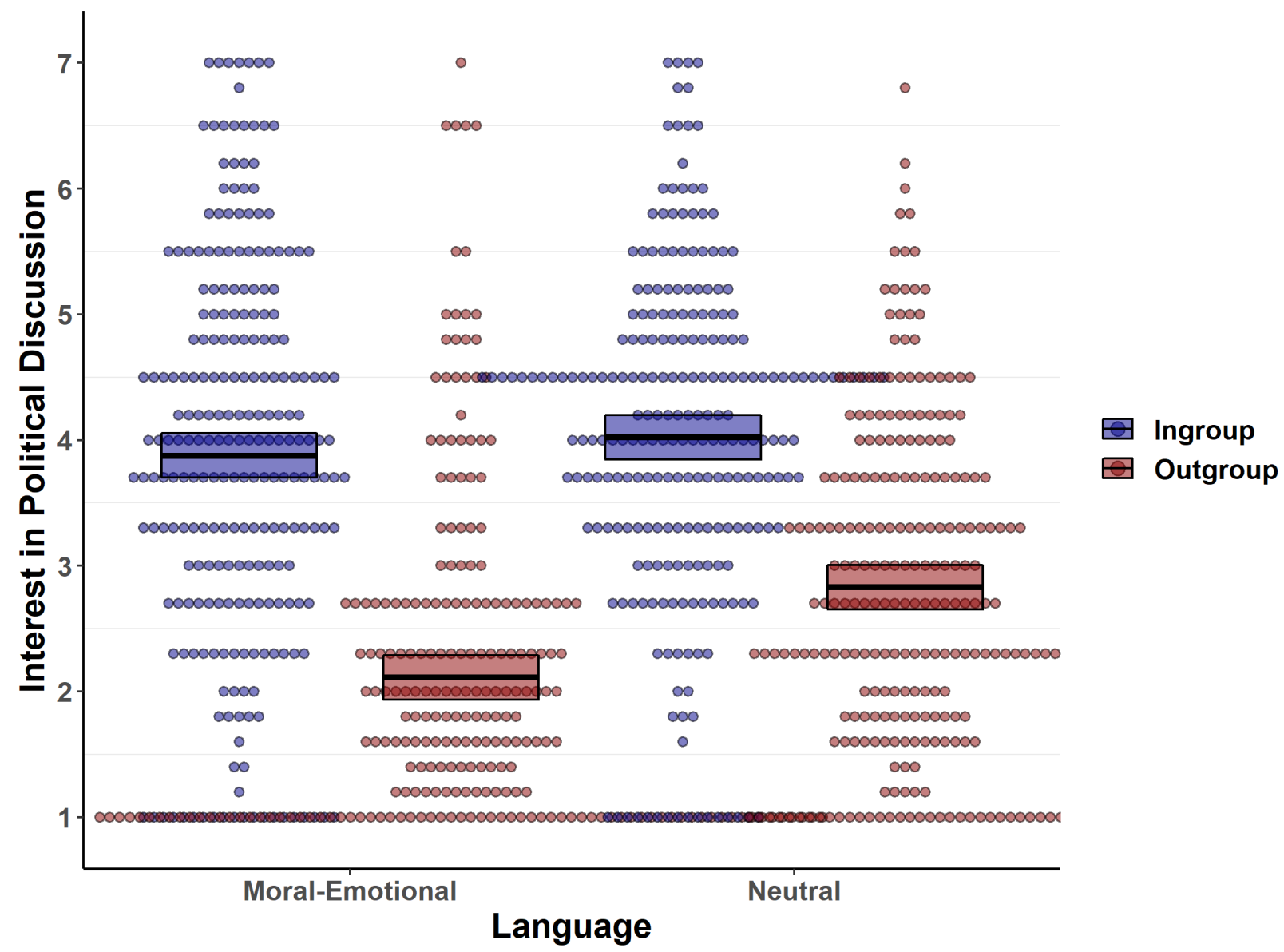

Fig. 8. The effect of language type and group on interest in having a political discussion with the message author. Participants were less interested having a political discussion with political outgroup members who expressed moral-emotional language compared to neutral language, but this was not true for ingroup members.

The results of Study 4 confirmed each of hypotheses FO1-3. These findings provide evidence that expressions of negative moral emotions that sanction the political outgroup can serve group identity functions such as making the expresser appear committed to their group via group identification strength. At the same time, however, these same expressions led to the perception that outgroup members were more close- 
minded and participants were less interested in having a political discussion with them. It is noteworthy that this double standard did not apply to the same degree to ingroup members who used the same language. Thus, people believe that outgroup members who use this language are not only highly identified but also selectively close minded.

\section{Discussion}

Across three preregistered experiments, we found that manipulating social media messages to contain moral-emotional language increased intentions to share the message among social media users. This finding highlights the causal impact of moralemotional language, and replicates in a controlled, experimental setting the phenomenon of 'moral contagion' observed in large-scale observational Twitter studies. The tendency to share moral-emotional messages is a function of individuals' political identity strength: strongly identified Democrats and Republicans are significantly more likely to share moral-emotional messages in the context of politics. In fact, individuals who were the most strongly identified in our sample reported were predicted to share moral-emotional messages (including more extreme messages in Study 3) about $80 \%$ of the time. This highlights the importance of social identity in explaining antecedents of moral contagion in terms of stable, individual differences in identity strength.

We did not find evidence that one-shot exposure to threatening or affirming political information causes individuals to share more moral-emotional messages. Our manipulation may have had difficulty making political identity salient because we recruited social media users only. Social media users are inundated with political content constantly, which may limit the impact of one-shot exposure to a news article in terms of making group identity more salient. Furthermore, social media users are known 
to be more politically active than the average population (Mellon \& Prosser, 2017), which also may limit the impact of reading a single political news article in terms of making group identity more salient. Future research is required to better understand the specific contexts that can impact political identity salience over and above enduring effects of individual differences in political identity strength.

Regarding functional outcomes, we found that people who share moral-emotional messages are viewed as more strongly identified with their ingroup. This finding suggests that expressing moral emotions can directly signal group identity strength to ingroup members, which is likely to help fulfil group belonging goals and contribute to being viewed as a committed group member. Combined with the ability of moral emotions to signal trustworthiness more broadly (Jordan \& Rand, 2019), the identitysignaling ability of moral emotions make them a powerful communicative tool to bind group members together in online networks.

We also found that expressing moral emotions lead the author to be perceived by outgroup members as less open-minded and less worthy of a political conversation. This finding highlights the double-edged sword of negative moral emotion expression in online networks: they can be used to express identity in ways that can enhance ingroup perceptions, but this comes at a cost for outgroup relations. This dilemma dovetails with broader views of human morality as a social mechanism that "binds and blinds" (Haidt, 2012). In an era of rising polarization, our results suggest that the spread of negative moral emotions contribute to polarized communications online. The spread of negative moral emotions can paint a picture of a social networks full of strongly identified group members who also strongly dislike outgroup members whether or not individuals are 
actually experiencing the emotions they expressing when they share or post them (Brady et al., 2020, 2021). This situation can contribute to 'false polarization' in which partisans become more polarized because they think others are more polarized than they really are (Finkel et al., 2020; Lees \& Cikara, 2020; Levendusky \& Malhotra, 2016; Wilson et al., 2020).

It is important to consider the boundary conditions of this causal effect. Our message stimuli were about contentious political topics in American politics, and thus it is unclear to what extent moral-emotional language impacts sharing beyond these political contexts. Furthermore, our conclusions are limited to American social media users and the American political context, however recent work has replicated the association between moral-emotional language and sharing in South American social media (Ariztia, 2020), suggesting that the effect may extend to non-American political contexts. Cross-cultural research comparing effects in different political contexts can enable broader conclusions about the generalizability of moral contagion online.

Our studies also had several limitations. We focused on the sharing of negative moral emotions such as outrage messages that sanctioned the outgroup. It is possible that positive moral emotions expressions such as awe and elevation (Keltner \& Haidt, 2003) could spread just as virally as negative emotions but have less costs for outgroup communication. Future research is required to test the group-level benefits of positive moral emotion expressions and contexts of moral praise rather than blame. One methodological limitation is that all of our stimuli expressed attitudes that corresponded to the attitudes aligned with ideologies most associated with Democrat and Republican parties. Although political party and political ideology are more correlated than ever 
before (Abramowitz \& Saunders, 2006), this approach may gloss over meaningful individual-level variation in attitudes that could impact the likelihood of choosing to share moral-emotional vs. neutral messages. Although we find that our results hold when adjusting for individual attitudes, it is important to test whether group identification explains the tendency to share moral-emotional content when an individual has attitudes that depart from the political party in some political issues. Finally, we note that we focused on political group identity in these studies, but people hold multiple group identities that can interact with political identity and potentially shape decisions to share moral-emotional content in varying ways. Ultimately, to fully understand moral contagion online, it is important to consider boundary conditions of specific social networks that contain varying group identities and social norms of communication (Brady et al., 2020).

\section{Conclusion}

In the context of contentious political conversations online, moral-emotional language causes political partisans to share the message more often, and that this effect was strongest in strong group identifiers. Expressing negative moral-emotional language in social media messages makes the message author appear more strongly identified with their group, but also makes outgroup members think the author is less open-minded and less worth of conversation. This work sheds light on antecedents and functional outcomes of moral-emotion expression in the digital age, which is becoming increasingly important to study as intergroup affective phenomena such as viral outrage and affective polarization are reaching historic levels. 


\section{References}

Abramowitz, A. I., \& Saunders, K. L. (2006). Exploring the Bases of Partisanship in the American Electorate: Social Identity vs. Ideology. Political Research Quarterly, 59(2), 175-187. https://doi.org/10.1177/106591290605900201

Abrams, D., \& Hogg, M. A. (2004). Metatheory: Lessons from social identity research. Personality and Social Psychology Review, 8(2), 98-106. https://doi.org/10.1207/s15327957pspr0802_2

Allcott, H., Braghieri, L., Eichmeyer, S., \& Gentzkow, M. (2020). The Welfare Effects of Social Media. American Economic Review, 110(3), 629-676. https://doi.org/10.1257/aer.20190658

Ariztia, F. de P. D. (2020). Contagio Moral en Redes Sociales: Cómo la Emoción Moral Transmite Mensajes y Polariza Diferentes Tipos de Conversaciones en Twitter. Pontificia Universidad Catolica de Chile (Chile).

Bail, C. A., Argyle, L. P., Brown, T. W., Bumpus, J. P., Chen, H., Hunzaker, M. B. F., Lee, J., Mann, M., Merhout, F., \& Volfovsky, A. (2018). Exposure to opposing views on social media can increase political polarization. Proceedings of the National Academy of Sciences, 115(37), 9216-9221. https://doi.org/10.1073/pnas.1804840115

Barberá, P., Jost, J. T., Nagler, J., Tucker, J. A., \& Bonneau, R. (2015). Tweeting From Left to Right: Is Online Political Communication More Than an Echo Chamber? Psychological Science, 26(10), 1531-1542. 
Brady, W. J., Crockett, M. J., \& Van Bavel, J. J. (2020). The MAD Model of Moral Contagion: The role of motivation, attention and design in the spread of moralized content online. Perspectives on Psychological Sciences. https://doi.org/10.31234/osf.io/pz9g6

Brady, W. J., \& McLoughlin, K. (2021). Theory-driven Measurement of Emotion (Expressions) in Social Media Text. In M. Dehghani \& R. Boyd (Eds.), The Atlas of Language Analysis in Psychology. Guilford Press.

Brady, W. J., McLoughlin, K., Doan, T. N., \& Crockett, M. (2021). How social learning amplifies moral outrage expression in online social networks.

Brady, W. J., Wills, J. A., Burkart, D., Jost, J. T., \& Van Bavel, J. J. (2019). An Ideological Asymmetry in the Diffusion of Moralized Content on Social Media Among Political Leaders. Journal of Experimental Psychology: General, 148(10), 1802-1813. https://doi.org/10.1037/xge0000532

Brady, W. J., Wills, J. A., Jost, J. T., Tucker, J. A., \& Van Bavel, J. J. (2017). Emotion shapes the diffusion of moralized content in social networks. Proceedings of the National Academy of Sciences, 114(28), 7313-7318. https://doi.org/10.1073/pnas.1618923114

Brewer, M. B. (1979). In-group bias in the minimal intergroup situation: A cognitivemotivational analysis. Psychological Bulletin, 86(2), 307-324. https://doi.org/10.1037/0033-2909.86.2.307

Crockett, M. J. (2017). Moral outrage in the digital age. Nature Human Behaviour, 1(11), 769-771. https://doi.org/10.1038/s41562-017-0213-3 
Duggan, M., \& Smith, A. (2016). The Political Environment on Social Media. Pew Research Center. http://www.pewinternet.org/2016/10/25/political-content-onsocial-media/

Finkel, E. J., Bail, C. A., Cikara, M., Ditto, P. H., lyengar, S., Klar, S., Mason, L., McGrath, M. C., Nyhan, B., Rand, D. G., Skitka, L. J., Tucker, J. A., Van Bavel, J. J., Wang, C. S., \& Druckman, J. N. (2020). Political sectarianism in America: A poisonous cocktail of othering, aversion, and moralization poses a threat to democracy. Science, 370(6516), 533-536. https://doi.org/10.1126/SCIENCE.ABE1715

Haidt, J. (2012). The righteous mind: Why good people are divided by politics and religion. Vintage.

Hopp, F. R., Fisher, J. T., Cornell, D., Huskey, R., \& Weber, R. (2020). The extended Moral Foundations Dictionary (eMFD): Development and applications of a crowdsourced approach to extracting moral intuitions from text. Behavior Research Methods, 1-15. https://doi.org/10.3758/s13428-020-01433-0

lyengar, S., Lelkes, Y., Levendusky, M., Malhotra, N., \& Westwood, S. J. (2019). The Origins and Consequences of Affective Polarization in the United States. Annual Review of Political Science, 22(1), 129-146. https://doi.org/10.1146/annurevpolisci-051117-073034

Jetten, J., Branscombe, N. R., Schmitt, M. T., \& Spears, R. (2001). Rebels with a cause: Group identification as a response to perceived discrimination from the mainstream. Personality and Social Psychology Bulletin, 27(9), 1204-1213. https://doi.org/10.1177/0146167201279012 
Jordan, J. J., \& Rand, D. G. (2019). Signaling When No One Is Watching: A Reputation Heuristics Account of Outrage and Punishment In One- Shot Anonymous Interactions. Journal of Personality and Social Psychology. https://doi.org/10.1037/pspi0000186

Judd, C. M., Westfall, J., \& Kenny, D. A. (2012). Treating stimuli as a random factor in social psychology: A new and comprehensive solution to a pervasive but largely ignored problem. Journal of Personality and Social Psychology, 103(1), 54-69. https://doi.org/10.1037/a0028347

Keltner, D., \& Haidt, J. (2003). Approaching awe, a moral, spiritual, and aesthetic emotion. In Cognition \& Emotion (Vol. 17, Issue 2, pp. 297-314).

Lazer, D. M. J., Baum, M. A., Benkler, Y., Berinsky, A. J., Greenhill, K. M., Menczer, F., Metzger, M. J., Nyhan, B., Pennycook, G., Rothschild, D., Schudson, M., Sloman, S. A., Sunstein, C. R., Thorson, E. A., Watts, D. J., \& Zittrain, J. L. (2018). The science of fake news. Science, 359(6380), 1094-1096. https://doi.org/10.1126/science.aao2998

Lees, J. M., \& Cikara, M. (2020). Understanding and Combating Misperceived Polarization [Preprint]. PsyArXiv. https://doi.org/10.31234/osf.io/ncwez

Levendusky, M. S., \& Malhotra, N. (2016). (Mis)perceptions of partisan polarization in the American public. Public Opinion Quarterly, 80(S1), 378-391. https://doi.org/10.1093/poq/nfv045

McEvoy, J. (2021). Capitol Attack Was Planned Openly Online For Weeks-Police Still Weren't Ready. Forbes. 
https://www.forbes.com/sites/jemimamcevoy/2021/01/07/capitol-attack-wasplanned-openly-online-for-weeks-police-still-werent-ready/

Mellon, J., \& Prosser, C. (2017). Twitter and Facebook are not representative of the general population: Political attitudes and demographics of british social media users. Research and Politics, 4(3), 1-9.

https://doi.org/10.1177/2053168017720008

Mooijman, M., Hoover, J., Lin, Y., Ji, H., \& Dehghani, M. (2018). Moralization in social networks and the emergence of violence during protests. Nature Human Behaviour, 2(6), 389-396. https://doi.org/10.1038/s41562-018-0353-0

Mosleh, M., Martel, C., Eckles, D., \& Rand, D. G. (2021). Shared partisanship dramatically increases social tie formation in a Twitter field experiment. Proceedings of the National Academy of Sciences, 118(7). https://doi.org/10.1073/pnas.2022761118

Mosleh, M., Pennycook, G., \& Rand, D. G. (2020). Self-reported willingness to share political news articles in online surveys correlates with actual sharing on Twitter. PLOS ONE, 15(2), e0228882. https://doi.org/10.1371/journal.pone.0228882

Postmes, T., Haslam, S. A., \& Jans, L. (2013). A single-item measure of social identification: Reliability, validity, and utility. British Journal of Social Psychology, 52(4), 597-617. https://doi.org/10.1111/bjso.12006

Rathje, S., Van Bavel, J. J., \& van der Linden, S. (2021). Outgroup animosity drives engagement on social media. Unpublished Manuscript. 
Rogers, N., \& Jones, J. J. (2021). Using Twitter Bios to Measure Changes in SelfIdentity: Are Americans Defining Themselves More Politically Over Time? Journal of Social Computing, 2(1), 1-13. https://doi.org/10.23919/JSC.2021.0002 Ronson, J. (2015). So You've Been Publicly Shamed. Penguin.

Tajfel, H., \& Turner, J. C. (1979). An Integrative Theory of Intergroup conflict. In W. G. Austin \& S. Worchel (Eds.), The Social Psychology of Intergroup Relations (pp. 33-47). Wadsworth. https://doi.org/10.1016/S0065-2601(05)37005-5

Turner, J C, Hogg, M. A., Oakes, P. J., Reicher, S. D., \& Wetherell, M. S. (1987). Rediscovering the Social Group: A Self-Categorization Theory. Blackwell. https://doi.org/10.1111/j.2044-8309.1987.tb00799.x

Turner, John C., Oakes, P. J., Haslam, S. A., \& McGarty, C. (2007). Self and Collective: Cognition and Social Context. Personality and Social Psychology Bulletin, 20(5), 454-463. https://doi.org/10.1177/0146167294205002

Valenzuela, S., Piña, M., \& Ramírez, J. (2017). Behavioral Effects of Framing on Social Media Users: How Conflict, Economic, Human Interest, and Morality Frames Drive News Sharing. Journal of Communication. https://doi.org/10.1111/jcom.12325

Wang, S.-Y. N., \& Inbar, Y. (2021). Moral-Language Use by U.S. Political Elites. Psychological Science, 32(1), 14-26. https://doi.org/10.1177/0956797620960397 Wilson, A. E., Parker, V., \& Feinberg, M. (2020). Polarization in the contemporary political and media landscape. In Current Opinion in Behavioral Sciences (Vol. 34, pp. 223-228). https://doi.org/10.1016/j.cobeha.2020.07.005 
Yarkoni, T. (2019). The Generalizability Crisis. PsyArXiv.

https://doi.org/10.31234/osf.io/jqw35 